Scharf, Philipp (2021): „Zur

Konstitution und Stabilität

von Milieus. Gegenkultur(en)

und Szenen in politischen

Transitionsphasen am

Fallbeispiel des "roten

Hannovers" In: Demokratie

Dialog 9 (2021) S. 30-36

doi:10.17875/gup2021-1771

\title{
Zur Konstitution und Stabilität von Milieus
}

\author{
Gegenkultur(en) und Szenen in \\ politischen Transitionsphasen \\ am Fallbeispiel des „roten \\ Hannovers"
}

Philipp Scharf

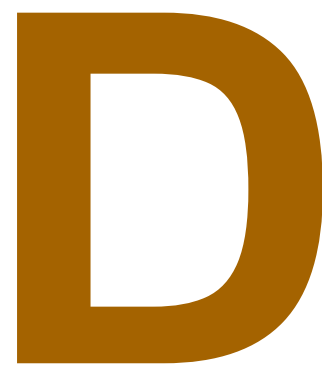

as "Superwahljahr 2019"1 sorgte deutschlandweit für einige Verwerfungen; neben der Europawahl waren die Bürger:innen aufgerufen vier Landesparlamen$\mathrm{te}^{2}$ neu zu wählen. Dabei verursachten insbesondere in den ostdeutschen Parlamenten die Etablierung der AfD sowie eine grundsätzlich abnehmende Parteienbindung des Wahlvolks eine erschwerte

1 Gabele, Julius: Superwahljahr 2019. Wahlen in Deutschland, in: Katapult-Magazin, URL: https://katapult-magazin.de/de/artikel/superwahljahr-2019 [eingesehen am 21.07.2021]

2 Gemeint sind Brandenburg, Bremen, Sachsen und Thüringen
Regierungsbildung. ${ }^{3}$ Doch auch in Niedersachsen fand eine - freilich überwiegend lokal beachtete, jedoch nicht minder spektakuläre - Wahl statt: Nach dem im Zuge der sogenannten „Rathausaffäre" erzwungenen Rücktritt des bis hierhin regierenden Oberbürgermeisters Stefan Schostok (SPD), gegen den Vorwürfe der schweren Veruntreuung erhoben wurden, musste in der niedersächsischen Landeshauptstadt Hannover ein neuer Oberbürgermeister gewählt werden. ${ }^{4}$

3 Vgl. Gabele

4 Vgl. Schinkel, Andreas/Doelke, Karl: Chronik eines schwierigen Abgangs, in: Hannoversche Allgemeine Zeitung, 30.04.2019, URL: https://www.haz.de/Hannover/Aus-der-Stadt/Rathausaffaere-Wie-die-SPD-ihren-Oberbuergermeister-ausgetrickst-hat [eingesehen am 21.07.2021]. 
Schwer wog also die Hypothek für die örtliche Sozialdemokratie, die seit 1946 durchgängig den Oberbürgermeister der Stadt stellte; schwerer noch wog das Wahlergebnis: Das "rote Rathaus" war grün geworden - ein absolutes Novum, das zu ungeahnten Verschiebungen in der „politischen Tektonik" ${ }^{15}$ der Leinestadt führte. So sparten einige journalistische Kommentatoren nicht mit Superlativen, demnach wurde Hannover "ein historisch bedeutsames Ereignis beschert. In der politischen Welt der Stadt ist seit diesem Sonntagabend nicht mehr viel so, wie es bisher war. ${ }^{\text {"6 }}$ Erste Stimmen interpretierten den Wahlausgang? als eine Abstrafung der hiesigen SPD, einerseits sollte ein phlegmatisches "Weiter so" der Stadtoberen demnach nicht weiter geduldet, andererseits die "Verfilzung von Politik und Verwaltung" beendet werden. Daher mag es nur oberflächlich paradox anmuten, dass im Wahlkampf kaum unterschiedliche Positionen zwischen den jeweiligen Kandidaten ausgemacht werden konnten. ${ }^{9}$ Bisherige sicher geglaubte Gewissheiten wurden mit dem Wahlergebnis endgültig auf den Kopf ge-

5 Schinkel, Andreas: "Mia san mia”: Wie die CDU Hannover aus der Wahlniederlage Kraft ziehen will, in: Hannoversche Allgemeine Zeitung, 14.11.2019, URL: Stadt/Hannover-nach-der-Oberbuergermeisterwahl-CDU-Onay-bekommt-100-Tage-Schonzeit [eingesehen am 21.07.2021]

6 Brandt, Hendrik: Kommentar: Neue Chance für Hannover, in: Hannoversche Allgemeine Zeitung, 28.10.2019, URL: https://www.haz.de/Hannover/Aus-der-Stadt/ Kommentar-der-HAZ-zur-OB-Wahl-in-Hannover-Neue-Chance-fuer-Hannover [eingesehen am 21.07.2021].

7 Der Kandidat der Grünen, Belit Onay, konnte sich in der Stichwahl gegen den parteilosen Kandidaten der CDU, Eckhard Scholz, durchsetzen. SPD-Kandidat Marc Hansmann schaffte es hingegen nicht in die Stichwahl.

8 Brandt: Kommentar.

9 Vgl. Schinkel, Andreas: Das sind die fünf wichtigsten Erkenntnisse aus der Diskussion, in: Hannoversche Allgemeine Zeitung, 23.10.2019, URL: https://www.haz.de/ Hannover/Aus-der-Stadt/HAZ-Salon-zur-OB-WahlDas-sind-die-fuenf-wichtigsten-Erkenntnisse-ausder-Diskussion [eingesehen am 21.07.2021]. stellt, die traditionelle sozialdemokratische Prägung $^{10}$ der Stadt scheint nun nicht mehr in Stein gemeißelt. Dadurch ergeben sich viele Fragen, Unsicherheiten und unklare Zukunftsprognosen," weswegen die Landeshauptstadt besonders aus zweierlei Gründen für die sozialwissenschaftliche Exploration spannend wird: Erstens liegt die Vermutung nahe, dass es hier starke Milieuzusammenhänge gibt. Zweitens scheinen diese Milieus oder doch zumindest die elektorale Loyalität, die lange Zeit Ausdruck eines Milieubewusstseins war - zu erodieren. Diesen Überlegungen folgend, ist die jüngste Wahlniederlage der SPD nicht die Ursache, sondern Symptom einer sich verändernden sozialen Ordnung. Dieser Artikel versucht nun einen Ausblick zu geben auf die Frage: Wie organisiert sich anhand von sozialen Milieus, gegenkulturellen Strömungen und lokalen Szenen das gesellschaftliche Zusammenleben Hannovers?

\section{Erosion oder Stabilität der sozialen Verbände?}

Spätestens seit dem 1986 veröffentlichten Werk „Risikogesellschaft" des Soziologen Ulrich Beck wird wissenschaftlich die Frage nach dem Fortbestehen und dem Formwandel sozialer Klassenlagen neu gestellt. Beck beschreibt eine janusköpfige Gleichzeitigkeit moderner Industriegesellschaften: Einerseits profitierten sie von zunehmendem Reichtum, andererseits werde analog „ein Prozeß der Individualisierung und Diversifizierung von Lebenslagen und Lebensstilen in Gang gesetzt, der das Hierarchiemodell sozialer Klassen und Schichten unterläuft und in seinem Wirklichkeitsgehalt in Frage stellt." ${ }^{\prime 12}$ Als problematisch an dieser Tendenz identifiziert er die un-

10 Gardemin, Daniel: Kleinräumige Untersuchung der SPD-Wählerschaft - Wahlanalysen in Hannover von 1998 bis 2008, in: Geiling, Heiko (Hrsg.): Die Krise der SPD. Autoritäre oder partizipatorische Demokratie, Berlin 2010, S. 259-301, hier S. 264.

11 Vgl. etwa: Schinkel: „Mia san mia”.

12 Beck, Ulrich: Risikogesellschaft. Auf dem Weg in eine andere Moderne, Frankfurt am Main 2020 [1986], S. 122. 
gleiche Verteilung von gesamtgesellschaftlichen Vorteilen und individuellen Risiken. ${ }^{13}$ Hierdurch löse sich die bekannte Klassenstruktur auf, da es zu einer "Potenzierung der Risiken"14 komme, deren Abwehr selbst den materiell Wohlhabenden nicht mehr möglich sei: „Not ist nicht hierarchisch, Smog ist demokratisch." ${ }^{15}$ Politisch wie wissenschaftlich bargen diese Beobachtungen einige Sprengkraft und verdeutlichten eindrücklich das Problem, mit dem sich Gesellschaftstheoretiker:innen konfrontiert sehen: Dem Formwandel ihres Beobachtungsgegenstandes.

Eine alternative Konzeption gesellschaftlicher Ordnungsvorstellungen stellt der Begriff des "Milieus" dar, der im deutschsprachigen Raum von Rainer M. Lepsius geprägt wurde. ${ }^{16}$ Am Beispiel des deutschen Kaiserreiches arbeitet Lepsius insgesamt vier zugrundeliegende "sozialmoralische Milieus" heraus, die eng mit den jeweiligen Parteien verbunden waren - und 1933 nicht überlebten. An diese Überlegungen anschließend skizziert Karl Rohe seinen Milieubegriff; dieser zielt auf "unterschiedliche kulturelle Manifestationen, auf Lebensweise, Mentalität und Deutungskultur. Aber konstitutiv für den Begriff ist letztlich nicht eine bestimmte Denkweise, sondern eine bestimmte Lebensweise. Kultur als Lebensweise kann man nur mit anderen zusammen haben. Milieu ist deshalb stets ein soziales, nicht bloß mentales Phänomen, auch wenn die Grenzen zwischen Lebensweise und Mentalität fließend sind." "17 Gegenüber dem materiellen Klassenmodell, das den

13 Vgl. ebd., S. 25

14 Ebd., S. 47

15 Ebd., S. 48

16 Vgl. hier und folgend: Lepsius, Rainer M.: Parteiensystem und Sozialstruktur. Zum Problem der Demokratisierung der deutschen Gesellschaft, in: Ritter, Gerhard Albert (Hrsg.): Die deutschen Parteien vor 1918, Köln 1973, S. 56-80

17 Rohe, Karl: Wahlen und Wählertraditionen in Deutschland. Kulturelle Grundlagen deutscher Parteien und Parteiensysteme im 19. und 20. Jahrhundert, Frankfurt am Main 1992, S.19.
Überlegungen Becks zugrunde liegt, betont der Milieu-Begriff also symbolische und kollektive Faktoren der Vergemeinschaftung und scheint so zunächst geeignet, um den dynamischen gesellschaftlichen Wandlungsprozessen Rechnung zu tragen. ${ }^{18}$ Jedoch: Neuere Forschungsergebnisse legen die Interpretation nahe, dass nicht nur ökonomische Klassen, sondern auch soziale und politische Milieus erodieren. ${ }^{19}$ Somit liegt zunächst die Deutung nahe: „Die Privatsphäre ist anscheinend der einzige noch funktionierende integrative Sozialraum. ${ }^{20}$

Dem lässt sich die These der Stabilität der Milieus gegenüberstellen, welche die Wandlungsprozesse nicht als vollständige Auflösung des Althergebrachten versteht, sondern von einer gewissen Neuordnung ausgeht. Die "Wahlverwandtschaft des Habitus" ${ }^{21}$ bliebe demnach weiterhin der soziale Kompass, weiche soziale Faktoren lösten die harten sozio-ökonomischen Differenzierungsmerkmale ab. Die vielfach vorgebrachte Beobachtung der abnehmenden Parteibindung würde oftmals fehlgedeutet, sie stelle keine "Krise der Milieus" infolge ihrer Auflösung, sondern vielmehr eine "Krise der politischen Repräsentation"22 dar. Der hier verwendete Milieubegriff zielt also stärker auf lebenspraktisch-kulturelle Entwürfe, ist zugleich jedoch keinesfalls beliebig, setzt er doch ein "gewisses Maß an lebensbiographischer Konstanz, effektiver Gemeinschaftsbildung und sozialer Verbindlichkeit ${ }^{\prime \prime 2}$ voraus. So wird das Milieu zu einem politischen Kollektivierungszusammenhang, weil sich hier politisch Gleichgesinnte ver-

18 Vgl. Marg, Stine: Mitte in Deutschland. Zur Vermessung eines politischen Ortes, Bielefeld 2014, S. 43.

19 Vgl. Neugebauer, Gero: Politische Milieus in Deutschland. Die Studie der Friedrich-Ebert-Stiftung, Bonn 2007, S. 11.

20 Ebd., S. 134

21 Vester, Michael et al.: Soziale Milieus im gesellschaftlichen Strukturwandel. Zwischen Integration und Ausgrenzung, Köln 2015, S. 80

22 Vgl. ebd., S. 1.

23 Rohe, S. 21 
sammeln. ${ }^{24}$ Hieraus ergibt sich, dass der elektorale Abgesang der Sozialdemokratie eben nicht den Niedergang des hiermit verknüpften Milieus bedeuten muss und die sozialen Verbände durchaus noch über einige Stabilität verfügen können. Anders ausgedrückt: Soziale Verbindlichkeit, geteilte Normen und Werte können auch nach der Ablösung der Parteibindung noch Wertigkeit besitzen; nicht jede wahrgenommene Veränderung muss also als Indiz einer vielfach beschworenen Krise der abnehmenden gesellschaftlichen Bindungen infolge erodierender Milieus sein.

Milieus verfügen demnach über eigene Kulturen, zumindest jedoch über genuine Kulturfragmente. Der engere thematische Fokus dieses Artikels liegt nun auf denjenigen Personengruppen und -verbänden, deren kulturelle Prägungen im Gegensatz oder Konflikt mit der von ihnen identifizierten Mehrheitsgesellschaft stehen - also Mitglieder von Subkulturen. ${ }^{25}$ Als besondere Spielart, die sich insbesondere durch diese Frontstellung auszeichnet, prägte der Historiker Dieter Langewiesche 1979 den Begriff der "Gegenkultur", also von antagonistischen „Teil- oder Gruppenkulturen mit beschränkten Autonomieansprüchen“ innerhalb einer Gesellschaft. Am Beispiel der historischen Arbeiterbewegung macht er sein Verständnis von Gegenkultur deutlich, demnach richte sie sich "gegen den Verbindlichkeitsanspruch der dominanten Kultur, die von den Trägern der Gegenkultur gedeutet wird als eine Klassenkultur zur Aufrechterhaltung politisch gesellschaftlicher Verhältnisse, deren gänzliche Veränderung

24 Vgl. Neugebauer, S. 22.

25 Milton M. Gordon definiert Subkulturen, die mehr seien als die bloße Zusammensetzung ihrer Teile, als "a concept used here to refer to a sub-division of a national culture, composed of a combination of factorable social situations such as class status, ethnic background regional and rural or urban residence, and religious affiliation, but forming in their combination a functioning unity which has an integrated impact on the participating individual", siehe Gordon, Milton M.: The Concept of Sub-Culture and its Application, in: Social Forces, Jg 26 (1947), H. 1, S. 40-42, hier S. 40 das Ziel der Gegenkultur ist." ${ }^{26}$ Hieran knüpft die Überlegung an, dass es - im Sinne Luhmannscher Systemtheorie - verschiedene gesellschaftliche Teilbereiche gäbe, ${ }^{27}$ in denen verschiedene Gruppen um kulturelle Deutungsmacht konkurrieren könnten.

Wenn eine gesellschaftliche Minorität in einem dieser Teilbereiche zu einiger Autonomie und Autorität gekommen ist, so kann von einer "Szene" gesprochen werden. Sie stellt eine wesentlich kleinere organisationale Einheit als die Milieus dar und kann als ein individualistischer Eskapismus aus den gesellschaftlichen Normvorstellungen bewertet werden - anders als im Milieu ist die Suche nach "Gesinnungsfreunden" hier nicht zwangsläufig politischer Natur. Szenen sind thematisch, zeitlich und teilweise auch örtlich gebunden und bedürfen einer gewissen Selbststilisierung, damit sind sie - stärker als das Milieu - auf kommunikative (Selbst-)Erzeugung angewiesen. ${ }^{28}$

\section{Das „rote Hannover"}

Die hier vorgestellte Triangulation der Begriffe Milieu, (Gegen-)Kultur und Szene verspricht ein deutlich komplexeres Bild der politischen Landkarte Hannovers zu zeichnen, als es die bloße Fokussierung auf einzelne erklärende Faktoren, wie beispielsweise Wahlergebnisse, leisten kann. Unter anderem diesem Vorhaben widmete sich der Fachbereich Linksradikalismus in der im Erscheinen begriffenen Studie über die „Konstitutions-

26 Langewiesche, Dieter: Arbeiterkultur in Österreich: Aspekte, Tendenzen und Thesen, in: Ritter, Gerhard A. (Hrsg.): Arbeiterkultur, Königstein/Ts. 1979, S. 40-57, hier jeweils S. 40

27 Vgl. Langewiesche, Dieter: Liberalismus und Sozialismus. Gesellschaftsbilder, Zukunftsvisionen und Bildungskonzeptionen, Bonn 2003, S. 280-281.

28 Vgl. Hitzler, Ronald/Bucher, Thomas/Niederbacher, Arne: Leben in Szenen. Formen jugendlicher Vergemeinschaftung heute, Opladen 2001, S. 20-24 
faktoren des ,anderen Hannover'" ${ }^{29}$ deren zentrale Ergebnisse hier kurz dargestellt werden sollen.

Wie bereits eingangs skizziert, ist die politische Kultur Hannovers eng mit der Geschichte und dem Wirken der örtlichen SPD verbunden. Während des Kaiserreichs und der Weimarer Republik fristeten die Sozialdemokrat:innen noch ein Ghettodasein in der Arbeiterhochburg Linden, das erst in den 1920er Jahren durch die Stadt Hannover eingemeindet werden sollte. Aus dieser Situation heraus bildete sich eine besondere Lagermentalität, die sich in der Hannoveraner SPD durch ein besonderes Hierarchiebewusstsein, herausragende Parteidisziplin sowie einen an Sachpolitik, Ausgleich und Konfliktmoderation orientierten Politikstil auszeichnete. Sukzessive prägte dieser Politikstil nicht nur die lokale SPD, sondern auch die politische Kultur Hannovers als solche. Vor diesem Hintergrund ist auch der derzeitige Wechsel der Wähler:innengunst zu interpretieren: Zwar können noch weitere Gründe - beispielsweise der entsprechende Bundestrend der Parteien oder ökonomische Erklärungsfaktoren - diesbezüglich gewiss eine wichtige Rolle spielen, die Art und Weise des Politikwechsels, eben wenig konfrontativ, dafür betont sachlich, bleibt "typisch hannoversch". Paradox mag es daher anmuten, dass gerade die jüngsten Wahlerfolge der Grünen für eine Stabilität des (vormals) sozialdemokratischen Milieus sprechen, da die Wähler:innenwanderung innerhalb eines politischen Lagers stattfand.

Dass dieses harmonische Politikverständnis die Stadt generell charakterisiert, zeigt sich im integrativen Umgang mit der linksradikalen Szene vor

29 Hier und im Folgenden: Scharf, Philipp/Schenke, Julian/ Tappé, Luke: Konstitutionsfaktoren des „anderen Hannover". Politische Kultur und linksradikales Engagement in der niedersächsischen Landeshauptstadt, Göttingen 2021, im Erscheinen. Konkret war das Erkenntnisziel der Studie, die lokalkulturellen Kontextbedingungen sowie die zentralen Charakteristika und Entwicklungstendenzen der Hannoverschen linksradikalen Szene zu identifizieren, um schließlich analysieren zu können, inwieweit Linksradikalismus als Prägefaktor der lokalen politischen Kultur angesehen werden kann.
Ort. Zwar wird diese von den Sicherheitsbehörden als ein hochgradig gefährlicher Hotspot in Niedersachsen dargestellt, ${ }^{30}$ die Ergebnisse unserer Studie legen jedoch nahe, dass es sich hier um einen verfehlten Alarmismus handelt. Vielmehr scheint es ein eher sprunghaftes öffentliches Interesse am Linksradikalismus zu geben, das routinierte Wahrnehmungskonjunkturen durchläuft. Sprich Infolge von öffentlichkeitswirksamen Protestereignissen steigt das Interesse der städtischen Gesellschaft am politischen Phänomen, um anschließend wieder einer gewissen Gleichgültigkeit zu weichen. Dies mag einerseits daran liegen, dass die hiesige linksradikale Szene hinsichtlich ihrer thematischen Ausrichtung sowie ihrer aktivistischen Performanz im Bundesvergleich eher durchschnittlich wirkt. Andererseits, und dies ist die entscheidende Besonderheit, gelang es auch mit Hilfe der beschriebenen langfristigen politischen Prägungen -, die Szene in Hannover fest zu institutionalisieren und Konfliktverläufe zu ritualisieren.

Es ist offensichtlich, dass die wechselseitig vollzogenen Abgrenzungs- und Annäherungsprozesse zwischen Stadt und Szene zyklisch reproduziert und gleichzeitig auch eingehegt werden. Dadurch wird die radikale Linke in Hannover zumindest von einigen Teilen der Bevölkerung als legitimer Bündnispartner und bedeutender Bestandteil des lokalpolitischen „Hefeteigs” wahrgenommen. Dieser Kompromiss kann als Ausdruck der Beziehung der lokalen Milieus, Subkulturen und Szenen interpretiert werden: Innerhalb der gegenkulturellen Subkultur, die sich aus dem sozialdemokratischen Milieu rekrutiert, können sich um verschiedene zentrale Orte bestimmte gegenkulturelle Szenen formieren, welche lokale Deutungsmacht erlangen, gleichzeitig aber den hegemonialen Konsens nicht grundsätzlich gefährden. Hierdurch entsteht ein stabiles Gleichgewicht der Kulturangebote, das die Landeshauptstadt bis in die Gegenwart hinein charakterisiert.

30 Vgl. Niedersächsisches Ministerium für Inneres und Sport (Hrsg.): Verfassungsschutzbericht 2019, Hannover 2020, S. 139 und 143-145 
Welche Kulturangebote und Orte des gegenkulturellen Bewusstseins für die politische Landschaft Hannovers entscheidend wirkten, welche Protestzyklen und Konfliktaustragungsmodi sich in der Stadt etablierten und wie "rot" Hannover heute tatsächlich noch ist, können Sie in unserer Studie nachlesen.

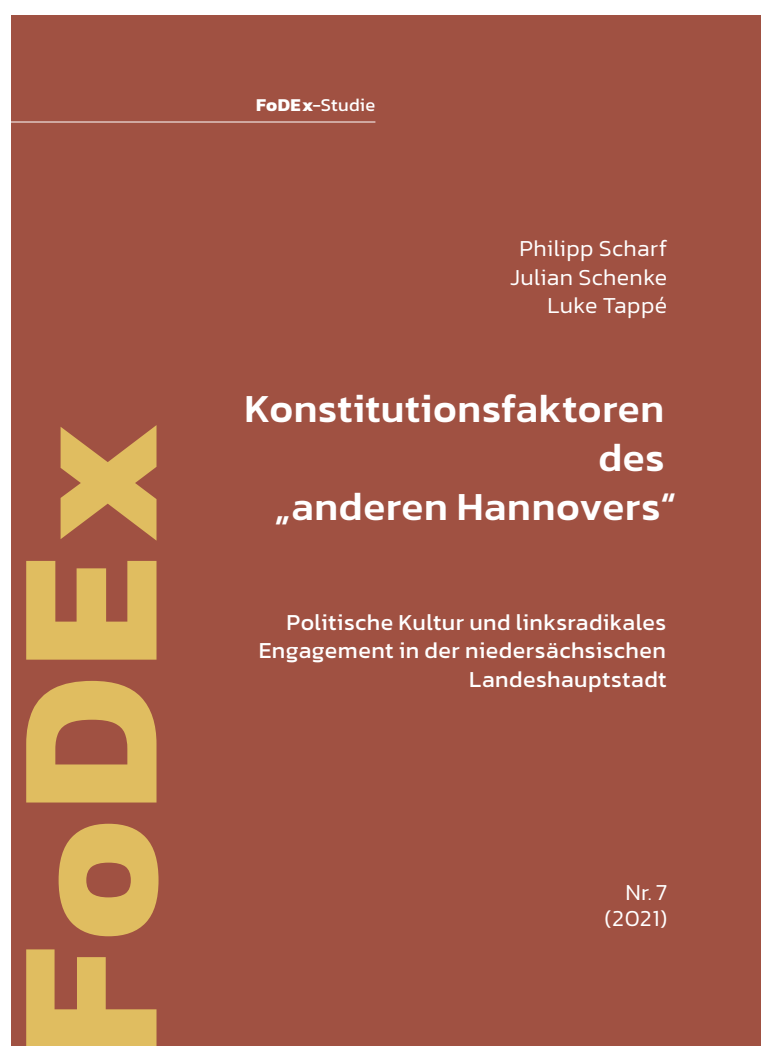

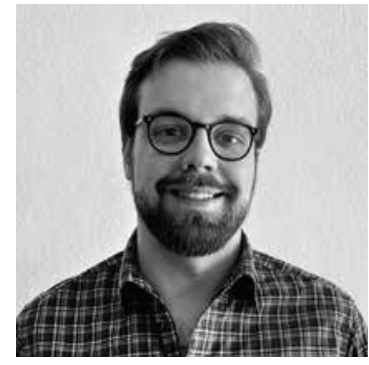

Philipp Scharf, M.A., ist Politikwissenschaftler und wissenschaftlicher Mitarbeiter am Institut für Demokratieforschung. Zu seinen Forschungsschwerpunkten zählen die Protest- und Bewegungsforschung sowie politische Kulturforschung. 


\section{Literatur:}

Beck, Ulrich: Risikogesellschaft. Auf dem Weg in eine andere Moderne, Frankfurt am Main 2020 [1986]

Brandt, Hendrik: Kommentar: Neue Chance für Hannover, in Hannoversche Allgemeine Zeitung, 28.10.2019, URL: https:// www.haz.de/Hannover/Aus-der-Stadt/Kommentar-der-HAZzur-OB-Wahl-in-Hannover-Neue-Chance-fuer-Hannover [eingesehen am 21.07.2021]

Gabele, Julius: Superwahljahr 2019. Wahlen in Deutschland, in Katapult-Magazin, URL: https://katapult-magazin.de/de/artikel/superwahljahr-2019 [eingesehen am 21.07.2021]

Gardemin, Daniel: Kleinräumige Untersuchung der SPD-Wählerschaft - Wahlanalysen in Hannover von 1998 bis 2008 in: Geiling, Heiko (Hrsg.): Die Krise der SPD. Autoritäre oder partizipatorische Demokratie, Berlin 2010, S. 259-301.

Gordon, Milton M.: The Concept of Sub-Culture and its Application, in: Social Forces, Jg. 26 (1947), H. 1, S. 40-42

Hitzler, Ronald/Bucher, Thomas/Niederbacher, Arne: Leben in Szenen. Formen jugendlicher Vergemeinschaftung heute, Opladen 2001

Langewiesche, Dieter: Arbeiterkultur in Österreich: Aspekte Tendenzen und Thesen, in: Ritter, Gerhard A. (Hrsg.): Arbeiterkultur, Königstein/Ts. 1979, S. 40-57.

Langewiesche, Dieter: Liberalismus und Sozialismus. Gesellschaftsbilder, Zukunftsvisionen und Bildungskonzeptionen, Bonn 2003, S. 280-281.

Lepsius, Rainer M.: Parteiensystem und Sozialstruktur. Zum Problem der Demokratisierung der deutschen Gesellschaft, in: Ritter, Gerhard Albert (Hrsg.): Die deutschen Parteien vor 1918 Köln 1973, S. 56-80
Marg, Stine: Mitte in Deutschland. Zur Vermessung eines politischen Ortes, Bielefeld 2014

Neugebauer, Gero: Politische Milieus in Deutschland. Die Studie der Friedrich-Ebert-Stiftung, Bonn 2007

Rohe, Karl: Wahlen und Wählertraditionen in Deutschland Kulturelle Grundlagen deutscher Parteien und Parteiensysteme im 19. und 20. Jahrhundert, Frankfurt am Main 1992.

Scharf, Philipp/Schenke, Julian/Tappé, Luke: Konstitutionsfaktoren des "anderen Hannover". Politische Kultur und linksradikales Engagement in der niedersächsischen Landeshauptstadt, Göttingen 2021 [im Erscheinen].

Schinkel, Andreas: Das sind die fünf wichtigsten Erkenntnisse aus der Diskussion, in: Hannoversche Allgemeine Zeitung, 23.10.2019, URL: https://www.haz.de/Hannover/Aus-derStadt/HAZ-Salon-zur-OB-Wahl-Das-sind-die-fuenf-wichtigsten-Erkenntnisse-aus-der-Diskussion [eingesehen am 21.07.2021]

Schinkel, Andreas: „Mia san mia“: Wie die CDU Hannover aus der Wahlniederlage Kraft ziehen will, in: Hannoversche Allgemeine Zeitung, 14.11.2019, URL: Stadt/Hannover-nach-der-Oberbuergermeisterwahl-CDU-Onay-bekommt-100-Tage-Schonzeit [eingesehen am 21.07.2021].

Schinkel, Andreas/Doelke, Karl: Chronik eines schwierigen Abgangs, in: Hannoversche Allgemeine Zeitung, 30.04.2019, URL: https://www.haz.de/Hannover/Aus-der-Stadt/Rathausaffaere-Wie-die-SPD-ihren-Oberbuergermeister-ausgetrickst-hat [eingesehen am 21.07.2021]

Vester, Michael/Oertzen, Peter von/Geiling, Heiko/Herrmann, Thomas/Müller, Dagmar: Soziale Milieus im gesellschaftlichen Strukturwandel. Zwischen Integration und Ausgrenzung, Köln 2015 . 